\title{
LINKAGE DISEQUILIBRIUM CAUSING SELECTION AT A NEUTRAL LOCUS IN POOLED TRIBOLIUM POPULATIONS
}

\author{
PETER STAM \\ Department of Genetics, Agricultural University, Wageningen, The Netherlands
}

Received 27.iii.74

\begin{abstract}
SUMmary
The role of linkage disequilibrium with respect to the changes in allele frequencies at a marker locus in newly started polymorphic populations is demonstrated using the marker locus black in Tribolium. The experiments, jointly taken, indicate that the black locus is selectively neutral, or nearly so, under the current experimental conditions.

A number of possible mechanisms for selection at the neutral marker locus, i.e. a single linked or non-linked locus and a number of linked and/or nonlinked loci through which selection acts, are discussed. On the basis of the experiments some of these models can be excluded, leaving three models for further evaluation: (A) a single linked fitness locus, (B) a number of linked fitness loci and $(C)$ a number of both linked and non-linked fitness loci.

The first of these models allows estimation of parameters (recombination fraction and selection coefficients). Models $B$ and $C$ are described in terms of heterozygosity of chromosome pairs. It is argued that in view of the history of a laboratory mutant stock, model $G$ fits best to the experimental results.
\end{abstract}

\section{INTRODUCTION}

A selectrvely neutral locus may be under indirect selection pressure because of the association of its alleles with those of non-neutral loci. This association (linkage disequilibrium involving a neutral locus) can be due to finite population size and/or recent hybridisation between groups. Therefore, apparent selection at a neutral locus is to be expected when a polymorphic laboratory stock (for a neutral marker locus) is initiated with population samples from different origin and with different genetic backgrounds.

As has repeatedly been pointed out (see e.g. Kimura, 1971; King and Dawson, 1972), linkage disequilibrium in newly started polymorphic populations might in part be responsible for the outcome of many experiments that at first sight indicate dominance, overdominance or frequency dependent selection at marker loci. It is therefore worth while to describe an experiment which clearly demonstrates the role that linkage disequilibrium may play with respect to the behaviour of marker alleles in a pooled population.

When introducing a mutant stock into a wild-type population it is to be expected that the mutant genotype is at a disadvantage since in general laboratory practice the construction of such a mutant stock from relatively small numbers of mutant individuals involves genetic drift, resulting in increased homozygosity in its genetic background.

In a pooled population, composed with individuals from both homozygous marker stocks (and possibly their $F_{1}$ ) the shifts in frequency of the marker alleles over generations can a priori be ascribed to:

(a) Frequency or non-frequency dependent selection at the marker locus itself. 
(b) Selection via a single linked locus.

(c) Selection via a number of linked loci.

(d) Selection via one or more non-linked loci.

(e) A combination of the possibilities $(a)$ to $(d)$, e.g. indirect selection through a number of both linked and non-linked loci (both groups of loci may contribute to linkage disequilibrium and therefore to differential fitness among the marker genotypes).

Experiments with Tribolium castaneum, using a wild-type and a mutant (black) laboratory stock, were started in order to investigate which of the mechanisms mentioned above governs the behaviour of the marker alleles in a population composed of individuals from each stock and their $F_{1}$. A classical $F_{2}$ was used to evaluate the role of non-linked loci with respect to the fitness contrast between the original marker stocks. For reasons of sequential inference, the order in which the $F_{2}$ experiment and the population experiment actually were carried out has been reversed in the presentation.

\section{EXPERIMENTS AND RESULTS}

Experiments were started with a wild-type and a homozygous black laboratory stock of the flour beetle Tribolium castaneum, to be denoted by CAW and $\mathrm{CAB}$, respectively. Black $(b)$ is an autosomal mutant affecting body colour, with intermediate expression in the heterozygote (Sokoloff, 1966). Both stocks were kindly provided by Dr A. Sokoloff, San Bernardino, California.

Populations were grown at $29^{\circ} \mathrm{C}$ and 70 per cent relative humidity on a standard medium of sifted whole wheat flour to which 5 per cent dried yeast was added.

\section{(i) $F_{2}$ experiment}

The stocks CAW and $\mathrm{CAB}$ were crossed in both directions to obtain $\mathrm{F}_{1}$ individuals $(+\mid b)$. Four replicate populations, each consisting of $200 \mathrm{~F}_{1}$ adults (sex ratio $1: 1$ ) were allowed to mate and lay eggs for 2 days in order to obtain an $\mathrm{F}_{2}$ generation. After this 2-day period the adults were removed from the medium.

If differential viability is an important component of differential fitness among the $F_{2}$ marker genotypes, this will result in a deviation from the $1: 2: 1$ ratio among the $F_{2}$ adults. Table 1 gives the observed numbers which in each of the four replicates closely fit to the expected numbers. This indicates absence of significant viability differences among $F_{2}$ marker genotypes. It also indicates that gametic selection probably played no role.

From a second set of four replicate $F_{2}$ populations the pupae were sexed, virgin adults were sampled and mixed in the exact $1: 2: 1$ ratio (100 adults of each sex per replicate) and were kept on fresh medium for a 2-day period to produce the next generation $\left(\mathrm{F}_{3}\right)$.

Since no viability differences among $F_{2}$ marker genotypes were found, no viability differences are expected in the $F_{3}$ generation either. The observed numbers of $\mathrm{F}_{3}$ adult marker genotypes are given in table 2. In none of the four replicates there is a significant deviation from Weinberg-Hardy frequencies. There is, however, a marked change in frequency of the 
$b$ allele: from 0.5 in $\mathrm{F}_{2}$ to an (unweighted) mean of 0.438 in $\mathrm{F}_{3}$. This change can now be ascribed to differential fertility among $F_{2}$ marker genotypes.

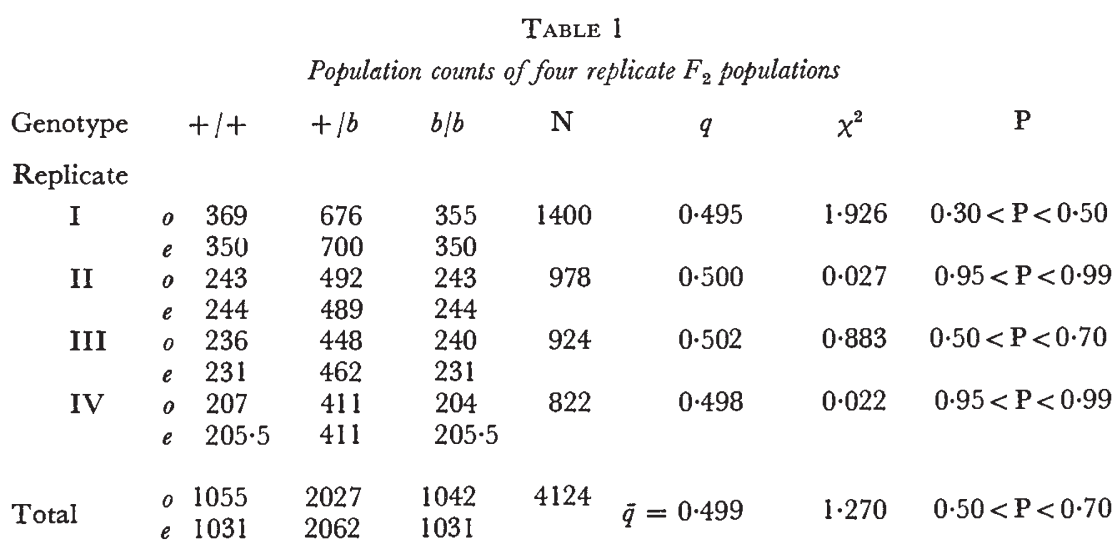

$o$ : observed number; $e$ : expected number on the basis of the $1: 2: 1$ ratio. $\mathrm{N}$ : sample size. The homogeneity chi-square $(6$ d.f.) is

$q$ : frequency of $b$ allele; $\bar{q}$ : unweighted mean of $q$.

$$
1.926+0.027+0.883+0.22-1.270=1.587(0.95<\mathrm{P}<0.99)
$$

In an $F_{2}$ there is approximate linkage equilibrium between marker locus and non-linked loci, and consequently only fitness loci on the marker chromosome will contribute to the fitness contrast among $\mathrm{F}_{2}$ marker genotypes. Thus, the results of the $\mathrm{F}_{2}$ experiment (i.e. existence of differential fertility among $\mathrm{F}_{2}$ marker genotypes) rule out the possibility that selection at the marker locus in a pooled population is exclusively due to non-linked loci (possibility $(d)$ mentioned in the Introduction).

TABLE 2

Population counts in the $F_{2}$ generation

$\begin{array}{cccccccc}\text { Genotype } & +1+ & +/ b & b / b & \mathrm{~N} & q & \chi^{2} & \mathrm{P} \\ \text { Replicate } & & & & & & & \\ \text { I } & 190 & 283 & 114 & 587 & 0.435 & 0.221 & 0.5<\mathrm{P}<0.7 \\ \text { II } & 243 & 430 & 180 & 853 & 0.463 & 0.159 & 0.5<\mathrm{P}<0.7 \\ \text { III } & 284 & 393 & 145 & 822 & 0.415 & 0.198 & 0.5<\mathrm{P}<0.7 \\ \text { IV } & 272 & 416 & 166 & 854 & 0.438 & 0.094 & 0.7<\mathrm{P}<0.8 \\ & & & & & \bar{q}=0.438 & & \end{array}$

Chi-square values represent values in the goodness-of-fit test to the Weinberg-Hardy frequencies. N: sample size; $q$ : frequency of $b$ allele; $\bar{q}$ : unweighted mean of $q$.

\section{(ii) Population experiment}

A number of populations were initiated each with 200 virgin adults (sex ratio $1: 1)$ from $\mathrm{CAW}, \mathrm{CAB}$ and their $\mathrm{F}_{1}$. The marker genotypes $(+/+,+/ b$ and $b / b$ ) were mixed in Weinberg-Hardy frequencies; five initial frequencies of the $b$ allele were chosen, viz.: $0 \cdot 07,0 \cdot 3,0.5,0 \cdot 7$ and 0.9 . With each initial frequency four replicate populations were started simultaneously. These $5 \times 4=20$ populations were maintained for eight generations. The adults with which each population was started were allowed to mate and lay eggs 
for 2 days, after which they were removed and discarded. After 35 days a random sample of approximately 500 adults of the next generation was extracted and transferred to fresh medium. After a 2-day stay these adults were removed, counted, classified and discarded. The latter cycle was repeated eight times. In this way generations were kept distinct and population size was kept approximately constant.

Notice that the conditions (including population density) during the first generation interval of this experiment and the $F_{2}$ experiment are identical, i.e. the results are fully comparable.

The result of this experiment is summarised in table 3 and fig. 1, where gene frequencies are plotted. From fig. 1 the following points are obvious:

TABLE 3

Data of the population experiment (frequencies of the $\mathrm{b}$ allele). Roman numerals indicate replicates

\begin{tabular}{|c|c|c|c|c|c|c|c|c|c|c|c|c|c|c|c|c|c|c|c|c|}
\hline \multirow[b]{2}{*}{ Generations } & \multicolumn{4}{|c|}{$q_{0}=\underbrace{0.07}$} & \multicolumn{4}{|c|}{$q_{0}=0.3$} & \multicolumn{4}{|c|}{$q_{0}=0.5$} & \multicolumn{4}{|c|}{$q_{0}=0.7$} & \multicolumn{4}{|c|}{$q_{0}=0.9$} \\
\hline & I & II & III & IV & I & II & III & IV & I & II & III & IV & I & II & III & IV & I & II & III & IV \\
\hline $\begin{array}{l}0 \\
1 \\
2 \\
3 \\
4 \\
5 \\
6 \\
7 \\
8\end{array}$ & $\begin{array}{l}0.06 \\
0.04 \\
0.03 \\
0.03 \\
0.03 \\
0.02 \\
0.02 \\
0.02 \\
0.02\end{array}$ & $\begin{array}{l}0.07 \\
0.05 \\
0.03 \\
0.03 \\
0.03 \\
0.02 \\
0.03 \\
0.02 \\
0.01\end{array}$ & $\begin{array}{l}0.07 \\
0.04 \\
0.03 \\
0.02 \\
0.01 \\
0.01 \\
0.01 \\
0.02 \\
0.02\end{array}$ & $\begin{array}{l}0.07 \\
0.05 \\
0.04 \\
0.03 \\
0.03 \\
0.03 \\
0.03 \\
0.03 \\
0.02\end{array}$ & $\begin{array}{l}0.30 \\
0.19 \\
0.13 \\
0.10 \\
0.08 \\
0.09 \\
0.08 \\
0.08 \\
0.08\end{array}$ & $\begin{array}{l}0.30 \\
0.21 \\
0.15 \\
0.12 \\
0.10 \\
0.09 \\
0.09 \\
0.09 \\
0.09\end{array}$ & $\begin{array}{l}0.30 \\
0.19 \\
0.14 \\
0.11 \\
0.10 \\
0.11 \\
0.10 \\
0.11 \\
0.11\end{array}$ & $\begin{array}{l}0.30 \\
0 \cdot 19 \\
0.15 \\
0.15 \\
0.12 \\
0 \cdot 14 \\
0.13 \\
0.12 \\
0.11\end{array}$ & $\begin{array}{l}0.34 \\
0 \cdot 26 \\
0 \cdot 24 \\
0 \cdot 21 \\
0 \cdot 19 \\
0.19 \\
0.18 \\
0.19\end{array}$ & $\begin{array}{l}0.50 \\
0.33 \\
0.26 \\
0.23 \\
0.23 \\
0.21 \\
0.19 \\
0.19 \\
0.18\end{array}$ & $\begin{array}{l}0.50 \\
0.34 \\
0.27 \\
0.26 \\
0.25 \\
0.22 \\
0.21 \\
0.19 \\
0.19\end{array}$ & $\begin{array}{l}0.50 \\
0.34 \\
0.26 \\
0.22 \\
0.20 \\
0.18 \\
0.17 \\
0.16\end{array}$ & $\begin{array}{l}0.70 \\
0.47 \\
0.39 \\
0.35 \\
0.32 \\
0.31 \\
0.29 \\
0.26 \\
0.28\end{array}$ & $\begin{array}{l}0.70 \\
0.48 \\
0.37 \\
0.35 \\
0.28 \\
0.28 \\
0.26 \\
0.25 \\
0.26\end{array}$ & $\begin{array}{l}0.70 \\
0.50 \\
0.40 \\
0.36 \\
0.35 \\
0.32 \\
0.35 \\
0.31 \\
0.33\end{array}$ & & $\begin{array}{l}0.90 \\
0.67 \\
0.59 \\
0.58 \\
0.52 \\
0.48 \\
0.45 \\
0.45 \\
0.44\end{array}$ & $\begin{array}{l}0.90 \\
0.66 \\
0.56 \\
0.52 \\
0.49 \\
0.48 \\
0.46 \\
0.49 \\
0.48\end{array}$ & $\begin{array}{l}0.90 \\
0.69 \\
0.63 \\
0.59 \\
0.57 \\
0.55 \\
0.54 \\
0.56 \\
0.54\end{array}$ & $\begin{array}{l}0.90 \\
0.66 \\
0.57 \\
0.54 \\
0.53 \\
0.52 \\
0.50 \\
0.53 \\
0.50\end{array}$ \\
\hline
\end{tabular}

1. During the first few generations there is a considerable selection pressure against the $b$ allele. Assuming a model in which selection operates through differential fertility, the joint data of the first-generation interval yield maximum likelihood estimates 0.74 and 0.12 for relative fertilities of $+/ b$ and $b / b$ respectively (relative to $+/+$ fertility) (the standard deviations of these estimates are 0.07 and 0.02 respectively). This gives an impression of the order of magnitude of the selection coefficients involved.

2. Selection pressure against the $b$ allele decreases as the populations breed.

3. After about eight generations the gene frequencies reach equilibria, the equilibrium gene frequency being dependent on the initial frequency.

These results can be taken to rule out the possibility that selection operates through the marker locus itself. If selection, be it frequency or non-frequency dependent, were acting through the marker locus, the ultimate result would be an equilibrium frequency which is the same in all populations. Of course, in a number of very specific situations (e.g. certain cases of non-random mating and selective interaction with other loci) selection at a given locus can give several equilibria, the particular one reached depending on the initial frequency. However, such a mechanism must be regarded improbable in the case at hand, since each of the five initial frequencies leads to a different equilibrium.

Fig. 1 suggests that the ultimate gene frequency in a population is also determined by its "history" and not only by its initial gene frequency. This point was checked as follows. In the fifth generation virgin adults were collected from the populations started with $q_{0}=0.9$ and $q_{0}=0.7$; with these individuals new initial populations were set up such that the marker genotypes were in Weinberg-Hardy frequencies with ${q_{0}}^{\prime}=0.8$ and $q_{0}{ }^{\prime}=0.6$, respectively. The gene frequencies in successive generations of these 


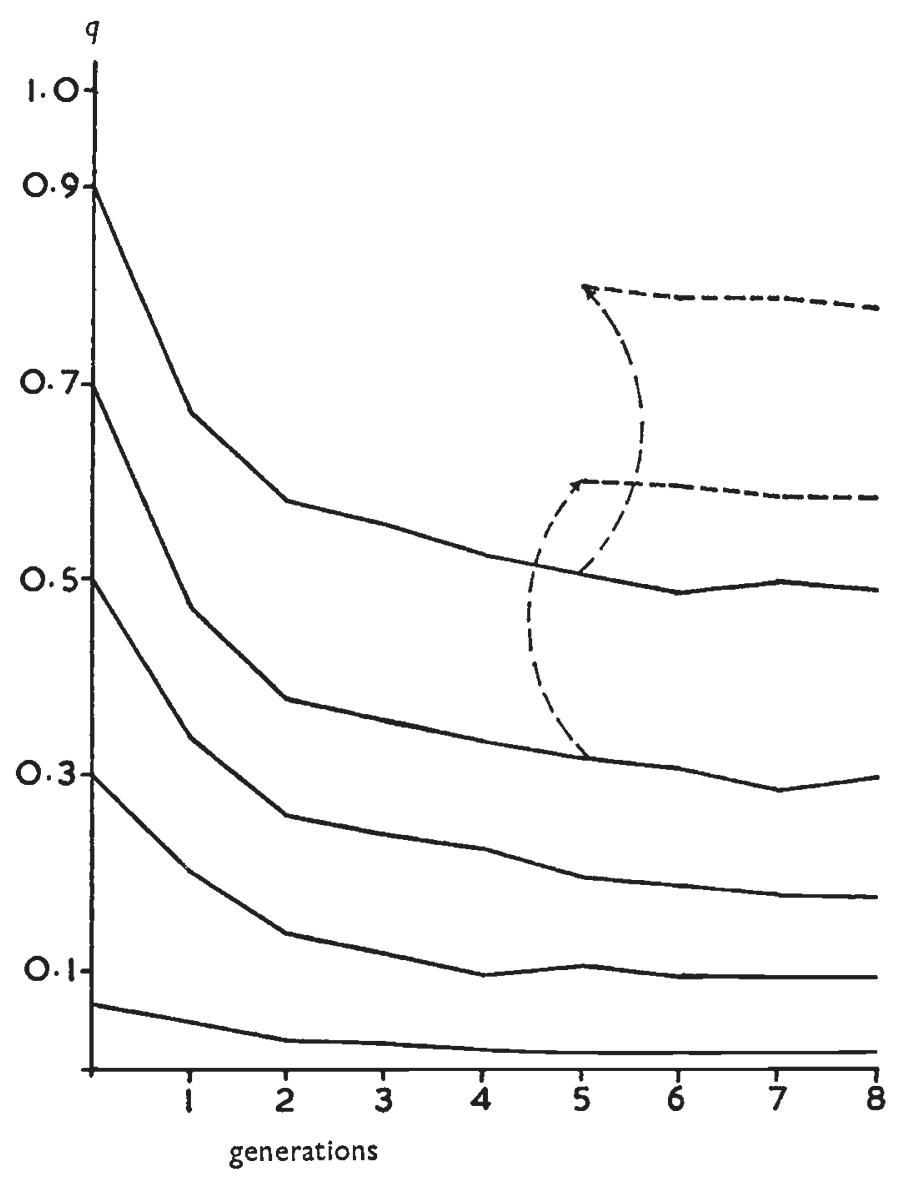

FIG. 1.-Frequency of $b$ allele $(q)$ in successive generations of the population experiment. Arrows and corresponding dotted lines indicate frequency of $b$ allele in re-started populations.

populations are given by the dotted curves of fig. 1. The result is unambiguous: the gene frequencies remain nearly constant, as if the marker locus were fitness-neutral.

It is clear that the stocks CAW and CAB must have different genotypic backgrounds contributing to the fitness contrast between the marker genotypes during the first generations of the experiment. Combining the results of the $\mathrm{F}_{2}$ experiment and the population experiment, the following conclusions can be drawn:

1. The marker locus is fitness-neutral (or nearly so) under the current experimental conditions.

2. Differential viability played no role in the process of changing gene frequencies. (The conclusion from the observed 1:2:1 ratio among $\mathrm{F}_{2}$ adults is supported by the fact that in only 5 out of the $5 \times 4 \times 8=160$ censuses of the populations a significant deviation from the Weinberg-Hardy frequencies, at the 5 per cent level of significance, was found.)

3. Indirect selection at the marker locus is not exclusively due to nonlinked fitness loci. 
This leaves us with the following models for indirect selection at the marker locus in the pooled populations:

A. Selection via a single linked locus.

B. Selection via a number of linked loci.

C. Selection via a number of both linked and non-linked loci.

In the following sections these models will be evaluated and discussed.

\section{Model A}

With this model, the estimates of relative fertilities of $+/ b$ and $b / b$, obtained from the data of the first-generation interval in the population experiment, are in fact estimates of the relative fertilities of genotypes at the fitness locus. These estimates can be used to obtain an estimate of the recombination frequency $(r)$ between marker and fitness locus. Table 4 gives the genotype distribution in an $\mathrm{F}_{2}$ together with relative fertilities and mean relative fertilities per marker genotype ( $A$ - $a$ denotes the fitness locus). Fertilities are expressed relative to $A a$ fertility for simplification of the algebra. Notice that the estimates 0.74 and 0.12 obtained earlier for fertilities of $A a$ and $a a$ relative to $A A$ fertility yield $s_{2}=-0.35$ and $s_{0}=0.84$.

\section{TABLE 4}

Genotype distribution in $F_{2}$ with relative fertilities and mean relative fertilities per marker genotype

$$
\begin{aligned}
& \text { Genotype Frequency } \begin{array}{l}
\text { Relative } \\
\text { fertility }
\end{array} \\
& \left.\begin{array}{lll}
\frac{+A}{+A} & \frac{1}{4}(1-r)^{2} & 1-s_{2} \\
\frac{+A}{+A} & \frac{1}{2} r(1-r) & 1 \\
\frac{+a}{+a} & \frac{1}{4} r^{2} & 1-s_{0}
\end{array}\right\} \quad \bar{w}_{++}=(1-r)^{2}\left(1-s_{2}\right)+2 r(1-r)+r^{2}\left(1-s_{0}\right) \\
& \left.\left.\begin{array}{lll}
\frac{+A}{b A} & \frac{1}{2} r(1-r) & 1-s_{2} \\
\frac{+A}{b a} \\
\frac{+a}{b A}
\end{array}\right\} \begin{array}{ll}
\frac{1}{2}\left[r^{2}+(1-r)^{2}\right] & 1 \\
\frac{+a}{b a} & \begin{array}{l}
\frac{1}{2} r(1-r) \\
1-s_{0}
\end{array}
\end{array}\right\} \begin{array}{l}
\bar{w}_{+b}=r(1-r)\left(1-s_{2}\right)+r^{2}+(1-r)^{2} \\
+r(1-r)\left(1-s_{0}\right)
\end{array} \\
& \left.\begin{array}{lll}
\frac{b A}{b A} & 1 r^{2} & 1-s_{2} \\
\frac{b A}{b a} & \frac{1}{2} r(1-r) & 1 \\
\frac{b a}{b a} & \frac{1}{4}(1-r)^{2} & 1-s_{0}
\end{array}\right\} \quad \bar{w}_{b b}=r^{2}\left(1-s_{2}\right)+2 r(1-r)+(1-r)^{2}\left(1-s_{0}\right)
\end{aligned}
$$

Starting with a population having genotype distribution $p^{2}(++)$, $2 p q(+b), q^{2}(b b)$ the frequency of the $b$ allele in the next generation $\left(q^{\prime}\right)$ is given by

$$
q^{\prime}=\frac{p q \bar{w}_{+b}+q^{2} \bar{w}_{b b}}{p^{2} \bar{w}_{++}+2 p q \bar{w}_{+b}+q^{2} \bar{w}_{b b}},
$$


where $\bar{w}$. . denotes the mean (relative) fertility of a given marker genotype. Substitution of $p=q=0.5$ and of the mean relative fertilities of marker genotypes given in table 4 into equation (1) yields

$$
q^{\prime}=\frac{r\left(1-s_{2}\right)+1+(1-r)\left(1-s_{0}\right)}{\left(1-s_{2}\right)+2+\left(1-s_{0}\right)}
$$

for the expected frequency of the $b$ allele in the $\mathrm{F}_{3}$ generation. So with $p=q=0.5$ the denominator of the right-hand side of equation (1) does not involve $r$, and one can express $r$ as a simple function of $q^{\prime}, s_{2}$ and $s_{0}$ :

$$
r=\frac{q^{\prime}\left(2-s_{2}\right)-p^{\prime}\left(2-s_{0}\right)}{\left(1-s_{2}\right)-\left(1-s_{0}\right)}\left(p^{\prime}+q^{\prime}=1\right) .
$$

For given values of $s_{2}$ and $s_{0}$, the maximum likelihood estimate of $r$ is given by equation (3) into which the maximum likelihood estimate of $q^{\prime}$ is substituted. Using $s_{2}=-0.35, s_{0}=0.84$ and $q^{\prime}=0.44$, equation (3) yields

$$
\hat{r}=0.323 \pm 0.018 \text {. }
$$

The parameters $s_{2}, s_{0}$ and $r$ can also be estimated simultaneously, of course, using the joint set of data of the first-generation interval of the population experiment and the $F_{2}$ experiment. This yields $\hat{s}_{2}=-0 \cdot 380$, $\hat{s}_{0}=0.841$ and $\hat{r}=0.321$, which hardly differs from the estimates obtained by the previous method. This is because the population experiment contains no information on $r$ whilst it provides by far the most information on $s_{2}$ and $s_{0}$, the $\mathrm{F}_{2}$ experiment contributing hardly any information on $s_{2}$ and $s_{0}$.

We now can use the set of estimated parameters, $\left(\hat{s}_{2}, \hat{s}_{0}, \hat{r}\right)=(-0.38$, $0.84,0.32)$, to compute the expected marker gene frequencies in further generations of the population experiment. These are shown in fig. 2 together with the observed gene frequencies. Fig. 2 shows that the estimated set of parameters of model A predict the result of the population experiment fairly well. The general trend of fig. 2 is that the predicted gene frequencies level off a little faster than the observed frequencies, indicating that the recombination frequency with model A probably has been overestimated.

\section{Models B and G}

Ascribing selection coefficients to a number of loci, one has to make assumptions on the distribution of these loci over the chromosomes and on their cumulative effect on fitness. With regard to the distribution of the relevant fitness loci one might assume a uniform distribution, i.e. the loci are distributed over the chromosomes without clusters or peaks in " density". This would be the most simple kind of distribution. However, taking into account the history of mutant stocks, it is likely that the relevant fitness loci, i.e. those loci which contribute to the fitness contrast between mutant and wild-type stock are concentrated in a region near the marker locus. This is because mutant stocks most often trace back to a small number of individual mutants. Repeated backcrossing of mutants to a wild-type stock (or other means of gene substitution) will result in substitution of the small sample of genes in the mutant by a larger sample from the wild-type stock, but alleles of loci closely linked to the marker locus have a smaller probability of being substituted than those of more loosely linked and unlinked loci. This will 


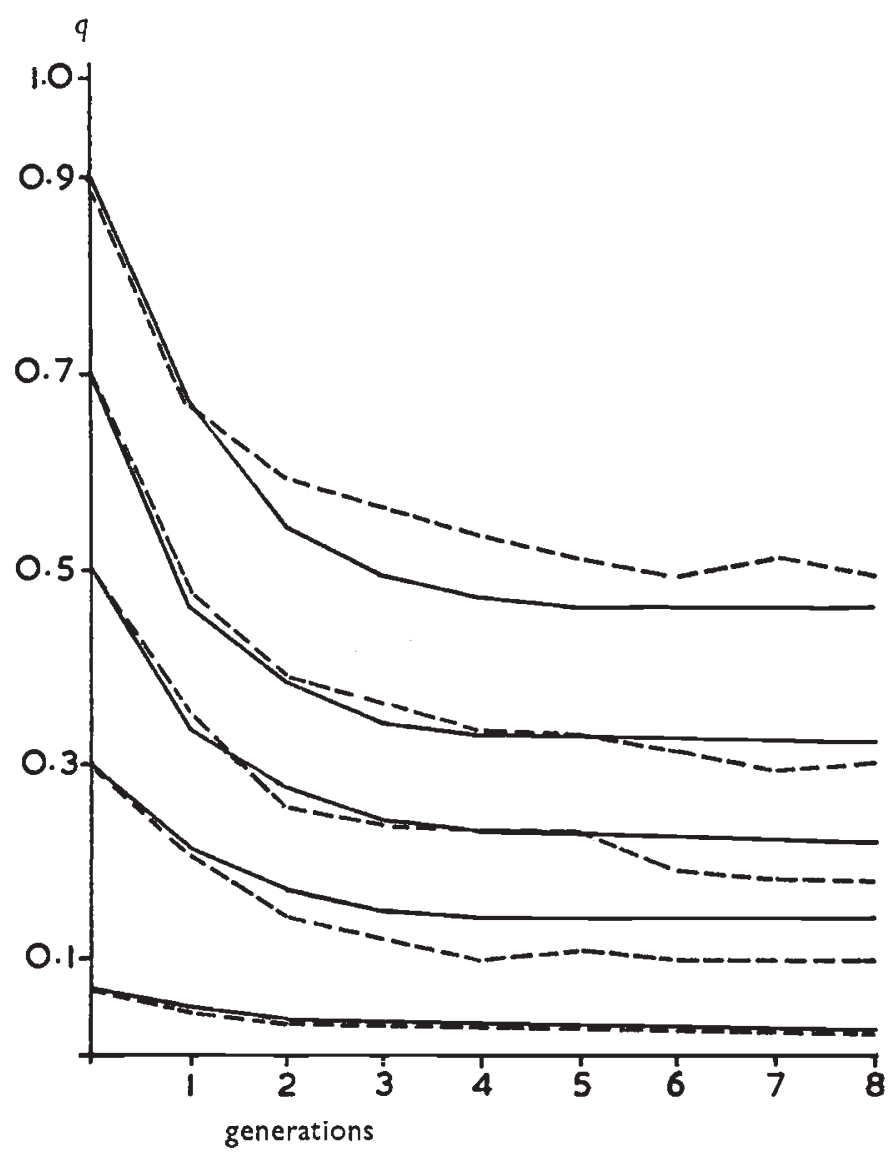

Fic. 2.-Frequency of $b$ allele ( $q$ ) in the population experiment (dotted lines) and frequencies predicted on the basis of parameter estimation for a single linked locus model (solid lines).

result in a decreasing homozygosity along the marker chromosome with increasing distance from the marker locus. The foundation of mutant stocks thus has two aspects of increased homozygosity (relative to the wild-type stock): (a) backcrossing only a small number of times causes a concentration of homozygosity in a region around the marker locus; $(b)$ backcrossing with small numbers causes increased overall homozygosity (distributed over the whole genome).

Taking into account these considerations, it might be expected that the loci contributing to the fitness contrast between $\mathrm{CAW}$ and $\mathrm{CAB}$ are distributed over the whole genome with a concentration around the marker locus.

Fitting models $\mathrm{B}$ and $\mathrm{C}$ to the data requires additional assumptions about a number of essentially unknown parameters and relationships: marker chromosome length, relative length of the marker chromosome, cumulative effect of multiple fitness loci and a relation between map distance and recombination frequency. For this reason fitting models $B$ and $G$ to the data makes little sense. 


\section{Discussion}

Formally the experiments provide no basis for distinction between a single locus model and a multiple locus model for indirect selection at the $b$ locus in the pooled populations. Bearing in mind the history of mutant stocks and the strong selective forces that must be ascribed to a single locus in the one-locus model, an explanation for the observed phenomenon in terms of multiple fitness loci or heterozygosity of chromosome segments is to be preferred above the single-locus model.

The distinction between the models is of course less important than the phenomenon of indirect selection at a neutral locus itself. The experiments clearly demonstrate the risk of ascribing selective forces to a neutral locus in early generations of a pooled population. In the case described here linkage disequilibrium is obvious because the wild-type and mutant stocks were not interbred to make them "isogenic" before the experiments were started. However, even when extracting homozygous strains from a population that has been polymorphic for many generations and which is in

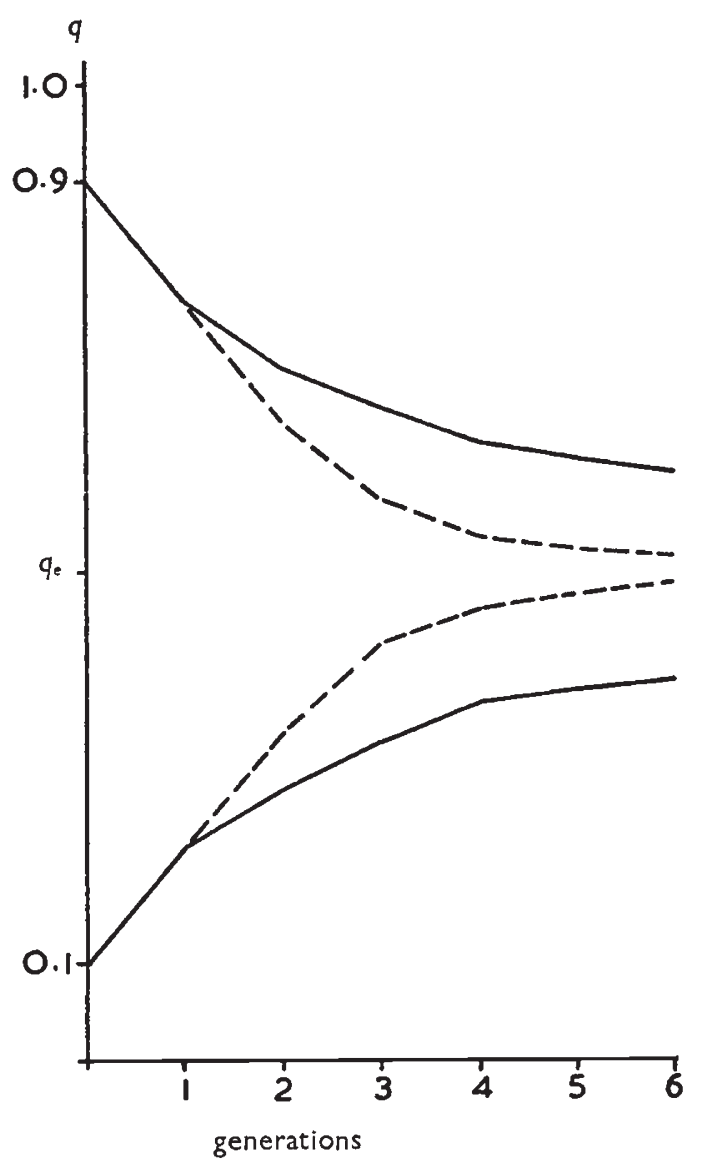

Frg. 3.-Comparison of gene frequency changes with constant selection coefficients (dotted lines) and with decreasing selection coefficients (solid lines) at an overdominant locus. $q_{e}$ : equilibrium gene frequency. 
approximate linkage equilibrium, there is a risk of introducing linkage disequilibrium when the homozygous strains are started with small samples. This has been pointed out by Ohta and Kimura (1969) and Kimura (1971). When two such homozygous marker strains are used to initiate a new polymorphic population, the expected pattern of apparent selective forces on the (neutral) marker locus is overdominance (see e.g. Ohta and Kimura, 1970). However, as the newly established population breeds, the association between the alleles of the marker locus and those of non-neutral loci will break down and consequently the apparent selection pressure at the marker locus decreases. In such a case the changes in marker gene frequency coincide with changes in apparent selection pressure and the phenomenon is easily mistaken to be the outcome of frequency dependent selection. Fig. 3 shows a hypothetical case of decreasing selective forces on an apparent overdominant locus. Since only two extreme initial gene frequencies are used in the example of fig. 3 , each gene frequency occurs only once in the course of the experiment. Experiments of this type therefore cannot be taken as proof of real frequency dependent selection, the latter being defined as a functional relationship between the frequency of a genotype and its fitness. In order to determine whether or not selection is really frequency dependent, genotype counts must be done within one-generation interval with a range of genotype and gene frequencies. Several examples of this approach can be found in literature (Sokal and Karten, 1964; Kojima and Yarbrough, 1967).

Acknowledgment.-I am grateful to professor J. H. van der Veen for advice on both the experimental part of the work and the presentation of the results.

\section{REFERENCES}

KIMURA, M. 1971. Theoretical foundations of population genetics at the molecular level. Theor. Pop. Biol., 2, 174-208.

kING, CH. E., AND DAWSON, P. s. 1972. Population biology and the Tribolium model. In Evolutionary Biology, vol. 5 (Ed. Th. Dobzhansky et al.). Appleton, Century, Crofts, New York.

KOJIMA, K. I., AND YARBROUGH, K. M. 1967. Frequency dependent selection at the esterase-6 locus in Drosophila melanogaster. Proc. Nat. Acad. Sci. U.S.A., 57, 645-649.

OHTA, T., AND KIMURA, M. 1969. Linkage disequilibrium due to random genetic drift. Genet. Res., 13, 47-55.

OHTA, T. AND KIMURA, M. 1970. Development of associative overdominance through linkage disequilibrium in finite populations. Genet. Res., 16, 165-177.

SOKAL, K. AND KARTEN, $\}$. 1964. Competition among genotypes in Tribolium castaneum at varying densities and gene frequencies (the black locus). Genetics, 49, 195-211.

sokolofr, A. 1966. The genetics of Tribolium and related species. Adv. Genet., suppl. 1. Acad. Press, New York. 